\title{
Potentially Inappropriate Medication Use in Multimorbid Elderly Inpatients: Differences Between the FORTA, PRISCUS and STOPP Ratings
}

\author{
Beate Wickop $^{1}$ (D) Steffen Härterich ${ }^{1} \cdot$ Christian Sommer $^{1} \cdot$ Anne Daubmann $^{2}$ • \\ Michael Baehr ${ }^{1}$ - Claudia Langebrake ${ }^{1,3}$
}

Published online: 1 August 2016

(C) The Author(s) 2016. This article is published with open access at Springerlink.com

\begin{abstract}
Background Several classifications to identify and avoid use of potentially inappropriate medications (PIMs) in the elderly have been published. To what extent these classifications match each other and whether there are differences in the prevalence of PIM use at admission, during the inpatient stay and at discharge are largely unreported.

Objectives To determine the PIM prevalence in elderly patients at a university hospital, with a special focus on different classification systems and the chronological sequence, and to examine a possible association between PIM use and the reason for admission, as well as severe side effects and consequences of PIM use during hospitalization.
\end{abstract}

Electronic supplementary material The online version of this article (doi:10.1007/s40801-016-0085-2) contains supplementary material, which is available to authorized users.

Beate Wickop

b.wickop@uke.de

1 Hospital Pharmacy, University Medical Centre Hamburg Eppendorf, Martinistrasse 52, 20246 Hamburg, Germany

2 Medical Biometry and Epidemiology, University Medical Centre Hamburg Eppendorf, Martinistrasse 52, 20246 Hamburg, Germany

3 Department for Stem Cell Transplantation, University Medical Centre Hamburg Eppendorf, Martinistrasse 52, 20246 Hamburg, Germany
Methods On the basis of the criteria provided by FORTA (Fit for the Aged), PRISCUS (Latin for 'time-honoured') and STOPP (Screening Tool of Older Persons' Potentially Inappropriate Prescriptions), medication in patients over the age of 65 years was screened retrospectively within four point prevalence analyses at admission, during the inpatient stay and at discharge. Evaluation of a possible association between PIM use and the primary diagnosis or severe side effects during hospitalization was performed according to an analysis using the World Health Organization Uppsala Monitoring Centre system for standardized case causality assessment.

Results Of 200 patients, $176(88 \%)$ received at least one PIM at admission, during the inpatient stay and/or at discharge (116 patients according to FORTA, 113 according to PRISCUS and 138 according to STOPP). When the PIM prevalence was compared between the three different sets of criteria, STOPP identified significantly more patients receiving PIMs than FORTA $(P=0.022)$ and PRISCUS $(P=0.010)$. At the patient level and at the drug level, the use of PIMs increased during the inpatient stay; however, the PIM prevalence was similar at admission and at discharge, both at the patient level and at the drug level.

Conclusion Medication is rated significantly differently by FORTA, PRISCUS and STOPP. In addition, a significant rise in prescribing of PIMs during the inpatient stay illustrates that a reduction in PIM use during the inpatient stay is essential, as it is known that avoiding PIM use in older adults is one strategy to decrease the risk of adverse events. 


\section{Key Points}

Three sets of criteria-FORTA (Fit for the Aged), PRISCUS (Latin for 'time-honoured') and STOPP (Screening Tool of Older Persons' Potentially Inappropriate Prescriptions) - rate drugs as potentially inappropriate medications (PIMs) quite differently. A comparison of FORTA, PRISCUS and STOPP showed that there are significant differences between them.

Especially during the inpatient stay, the prescribing of PIMs was shown to be high. As it is known that avoiding PIM use in older adults is one strategy to decrease the risk of adverse events, a reduction in PIM use during the inpatient stay is essential.

Polypharmacy has been identified as a risk factor for use of PIMs, so reducing the total number of prescribed drugs might be a starting point to reduce PIM use.

\section{Introduction}

Elderly people often suffer from multiple co-morbidities, which predispose them to potentially harmful polypharmacy and inappropriate prescribing. Therapy based on guidelines can be problematic and is rarely examined in clinical trials in elderly patients. In addition, alterations in pharmacokinetics and pharmacodynamics, due to the advanced age of patients, have to be considered [1]. As a result, the elderly are particularly vulnerable to certain drugs-e.g. long-acting benzodiazepines, which might result in prolonged sedation and increase the risk of falls [2]. Therefore, these drugs are classified as potentially inappropriate medications (PIMs) for the elderly because they carry an increased risk of adverse drug events and result in major patient safety concerns. Several classifications have been published to identify and avoid PIM use. In the USA, the Beers list is the prevailing one [3]. In Europe, several sets of criteria have been published. In our study, the following three screening tools were chosen to identify PIMs: FORTA (Fit for the Aged) [4], PRISCUS (Latin for 'time-honoured') [5] and STOPP (Screening Tool of Older Persons' Potentially Inappropriate Prescriptions) [6], because they have been shown to be suitable for the German pharmaceutical market.

In several studies, STOPP and the Beers criteria have been applied to detect PIM use [7-9]. One study has compared PRISCUS with Beers and STOPP [10], and in a recent study, the overlap between different sets of criteria was shown to be small [11].

However, to our knowledge, FORTA, PRISCUS and STOPP have not been applied to one patient collective simultaneously. The objective of the present study was to determine which PIMs are used by elderly patients at the University Medical Centre Hamburg Eppendorf (UKE) and how the prevalence of PIM use changes from admission over the course of the inpatient stay to discharge. In addition, a possible association between PIM use and the primary diagnosis/reason for admission, as well as severe adverse events and consequences of PIM use during hospitalization, were examined. This information was then used to develop a practical tool for minimizing PIM use.

\section{Methods}

\subsection{Setting and Participants}

The study was conducted at UKE, a university hospital with 1460 beds. On the basis of the criteria provided by FORTA, PRISCUS and STOPP, medication use in patients above the age of 65 years was screened retrospectively within four point prevalence analyses (distributed equally over one year in September 2011, December 2011, May 2012 and July 2012) at admission, during the inpatient stay and at discharge. Patients were included in the study if they were admitted via the emergency department and had at least five drugs prescribed at admission. Patients were not eligible if they were admitted because of a malignant disease or had undergone solid organ or stem cell transplantation.

Medication data were recorded using Anatomical Therapeutic Chemical (ATC) classification codes [12]. The reason for admission and all diagnoses were coded according to the International Statistical Classification Of Diseases And Related Health Problems, 10th revision, German Modification (ICD-10-GM), version 2012 [13].

Information on medication use at admission was extracted from the admission form in each electronic patient record (Soarian ${ }^{\circledR}$ Clinicals, Erlangen, Germany). If it was not recorded there, medication use at day 1 during the hospital stay was graded as medication use at admission, leaving out acute medication, such as antibiotics or analgesics. Information on medication use at discharge was extracted from the discharge letter. Information on all drugs used during the hospital stay could be obtained from the computerized physician order entry (CPOE) ATCHost ${ }^{\circledR}$ (Baxter, Utrecht, the Netherlands). Details of the medication were recorded in a database and correlated with the diagnoses and reason for admission. 


\subsection{Classification Systems for PIMs}

The FORTA drug classification system assigns positive and negative labels (ranging from class A to class D) to 190 of the substances most frequently used for long-term drug therapy in older patients, according to the state of evidence as to risk/benefit and age appropriateness. FORTA was validated in 2014 [14].

The PRISCUS list was created for the German pharmaceutical market on the basis of expert knowledge, using the Delphi technique. Eighty-three drugs in a total of 18 drug classes are rated as potentially inappropriate for elderly patients [5].

STOPP 2008 comprises 65 clinically significant criteria for potentially inappropriate prescribing in older people. The STOPP criteria are arranged according to the relevant physiological systems, for ease of use. Each criterion is accompanied by a concise explanation as to why the prescription is potentially inappropriate. Eighteen experts in geriatric pharmacotherapy were recruited to establish the content validity of STOPP by a Delphi consensus method [6].

\subsection{Data Analysis}

Statistical analyses were performed using Microsoft ${ }^{\circledR}$ Office Access 2010, Microsoft ${ }^{\circledR}$ Office Excel 2010 and SAS (Statistical Analysis System) version 9.4 (SAS Institute Inc., Cary, NC, USA). The two-sided significance level was set at $5 \%(P \leq 0.05)$.

The primary objectives of the study (which PIMs are used by elderly patients at UKE, and how the prevalence of PIM use changes from admission over the course of the inpatient stay to discharge) were evaluated using mixed logistic regression with the binary endpoint of PIM use (yes or no) and a mixed Poisson regression with the number of PIMs as the endpoint to examine statistically significant differences. PIM use was studied in total and separately on the basis of the FORTA, PRISCUS and STOPP criteria.

Subgroup analyses were performed concerning age, sex, previous residential situation, number of prescribed drugs, length of the hospital stay, and hospital stay in a surgical ward versus an internal medicine ward. Metric variables were dichotomized.

Evaluation of a possible association between PIM use and the primary diagnosis or severe side effects during hospitalization (secondary objectives) was performed according to an analysis using the World Health Organization Uppsala Monitoring Centre (WHO-UMC) system for standardized case causality assessment [15]. The causality assessment was performed independently by three pharmacists. Different ratings were discussed until a consensus was reached. Severe side effects were rated according to the US Food and Drug Administration definition of grade 3: severe or medically significant but not immediately life threatening; hospitalization or prolongation of hospitalization indicated; disabling; limiting selfcare activities of daily living.

To assess severe side effects and consequences of PIM use during hospitalization, all clinical records of the patients were checked, e.g. concerning documented falls.

\section{Results}

\subsection{Study Population}

The characteristics of the patients and the number of PIMs used per patient according to the FORTA, PRISCUS and STOPP criteria are presented in Table 1. In summary, 200 patients were included in the study, the average age was 78.8 years and $61 \%$ of the patients were female.

Table 1 Patient characteristics

\begin{tabular}{ll}
\hline Characteristics & Values \\
\hline Sex $(N)$ & \\
Female & 121 \\
Male & 79 \\
Age (years) & \\
Median & 79.0 (range 65-96) \\
Mean & 78.8 (SD 7.5) \\
Duration of inpatient stay (days) & \\
Median & 12.0 (range 3-28) \\
Mean & 13.1 (SD 6.2) \\
Number of drugs per patient & 18.0 (range 8-35) \\
Median & 18.6 (SD 5.5) \\
Mean & \\
Number of PIMs per patient & \\
Total & 4.0 (range 0-8) \\
Median & 2.3 (SD 1.8) \\
Mean & \\
FORTA & 2.5 (range 0-5) \\
Median & 1.0 (SD 1.1) \\
Mean & \\
PRISCUS & 2.5 (range 0-6) \\
Median & 1.1 (SD 1.0) \\
Mean & \\
STOPP & 1.4 (SD 1.4) \\
Median & \\
Mean & \\
\hline
\end{tabular}

FORTA (Fit for the Aged), PIM potentially inappropriate medication, PRISCUS [Latin for 'time-honoured'], SD standard deviation, STOPP (Screening Tool of Older Persons' Potentially Inappropriate Prescriptions) 
Table 2 Numbers of patients prescribed potentially inappropriate medications (PIMs), and numbers of PIMs prescribed per patient, during the study period

\begin{tabular}{lc}
\hline PIMs & Values \\
\hline PIMS per patient & \\
1 PIM & 55 \\
2 PIM & 44 \\
3 PIM & 30 \\
4 PIM & 26 \\
5 PIM & 8 \\
6 PIM & 7 \\
7 PIM & 4 \\
8 PIM & 2 \\
Total number of patients receiving PIMs & 176 \\
\hline
\end{tabular}

The most common reasons for hospitalization were fractures $(N=35)$, strokes $(N=12)$, left-ventricular heart failure $(N=11)$ and secondary right ventricular heart failure $(N=10)$.

Of 200 patients, $176(88 \%)$ received at least one PIM at admission, during the inpatient stay and/or at discharge. The median number of PIMs received by the patients was 4 , ranging from 0 (in $12 \%$ of patients) to 8 (in $1 \%$ of patients). Most patients received 1 PIM (in $28 \%$ ) or 2 PIMs (in $22 \%$ ) during the study period (see Table 2).

In total, 116 patients received FORTA PIMs, 113 patients received PRISCUS PIMs and 138 patients received STOPP PIMs. Eight patients received only FORTA PIMs, 19 patients received only PRISCUS PIMs and 23 patients received only STOPP PIMs. Eleven patients received FORTA + PRISCUS PIMs, 32 patients received FORTA + STOPP PIMs, 18 patients received PRISCUS + STOPP PIMs and 65 patients $(37 \%$ of all patients receiving PIMs) received FORTA + PRISCUS + STOPP PIMs (see Fig. 1).

\subsection{Primary Objectives}

Which PIMs are used by elderly patients at UKE, and how does the PIM prevalence change from admission to discharge?

The PIMs prescribed most often were zopiclone ( $N=63 ; \quad$ PRISCUS), acetylsalicylic acid $(N=23$; STOPP), lorazepam ( $N=22$; FORTA, PRISCUS and STOPP), amlodipine ( $N=21$; STOPP) and ibuprofen ( $N=20$; FORTA and STOPP). When grouped according to level 3 ATC codes, drugs from the group of hypnotics and sedatives were by far the most commonly used $(N=84)$.

At admission, 126 patients $(63 \%)$ received at least one PIM. During the inpatient stay, $168(84 \%)$ received at

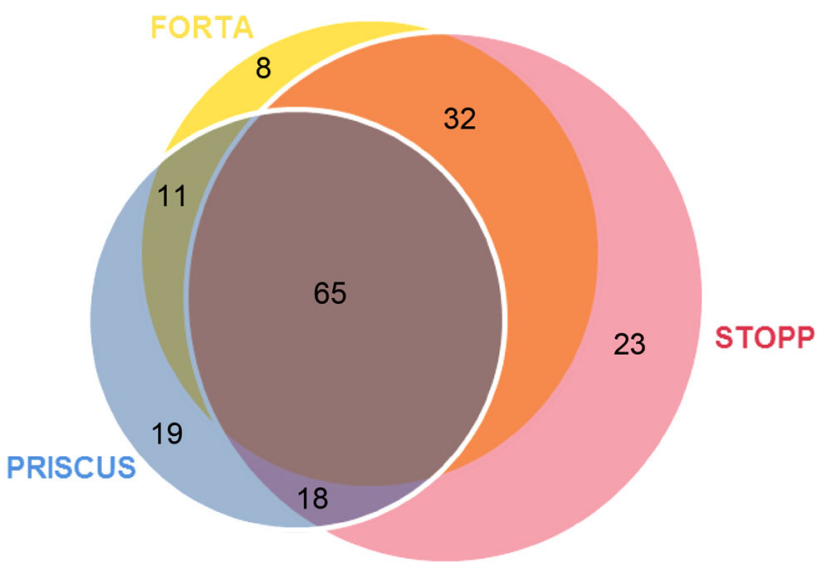

Fig. 1 Venn diagram showing the degrees of overlap between the FORTA, PRISCUS and STOPP tools in terms of potentially inappropriate medications (PIMs) identified; 65 patients (37\% of all patients receiving PIMs) received FORTA + PRISCUS + STOPP PIMs. FORTA (Fit for the Aged), PRISCUS [Latin for 'time-honoured'], STOPP (Screening Tool of Older Persons' Potentially Inappropriate Prescriptions)

least one PIM. At discharge, 126 (63\%) received at least one PIM. Multiple classifications were possible (see Fig. 2a).

Concerning drugs, the PIM proportion of the total number of medications was $12.5 \%$ (463 of 3717); $12.7 \%$ of drugs (223 of 1757) at admission, $11.9 \%$ (397 of 3324) during the inpatient stay and $10.5 \%$ (217 of 2068) at discharge were regarded as PIMs (see Fig. 2b).

To summarize, at the patient level and at the drug level, the numbers of PIMs rose during the inpatient stay. At the patient level, it could be shown that the differences were significant $(P<0.0001)$ between admission and the inpatient stay, as well as between the inpatient stay and discharge. At the drug level, the rise in the overall number of prescribed drugs during the hospital stay has to be considered. Nevertheless, the numbers of PIMs differed significantly between admission and the inpatient stay $(P=0.0087)$, as well as between the inpatient stay and discharge $(P=0.0091)$ (see Fig. 2a, b).

Within FORTA, PRISCUS and STOPP, significant changes could be found at the patient level from admission to the inpatient stay, as well as from the inpatient stay to discharge, concerning FORTA PIMs $(P=0.0071$ and $P=0.0007$, respectively) and PRISCUS PIMs (both $P<0.0001)$. For STOPP PIMs, the difference was significant only between admission and the inpatient stay $(P=0.0214)$. The difference between admission and discharge was not significant in any classification system. At the drug level, a significant difference in prescribing of PRISCUS PIMs could be shown between admission and the inpatient stay $(P=0.0060)$ and between the inpatient stay and discharge $(P=0.0005)$; for STOPP PIMs, a 
Fig. 2 Changes in the prevalence of potentially inappropriate medication (PIM) use from admission to discharge. FORTA (Fit for the Aged), PRISCUS [Latin for 'time-honoured'],

STOPP (Screening Tool of Older Persons' Potentially Inappropriate Prescriptions)

a Patient level

\begin{tabular}{llllll}
\hline & PIM (total) & FORTA & PRISCUS & STOPP \\
\hline At admission & 126 patients, thereof & $77(61 \%)$ & $45(36 \%)$ & $100(79 \%)$ \\
\hline Inpatient stay & 168 patients, thereof & $104(62 \%)$ & $104(62 \%)$ & $123(73 \%)$ \\
\hline At discharge & 126 patients, thereof & $70(56 \%)$ & $44(35 \%)$ & $107(85 \%)$
\end{tabular}

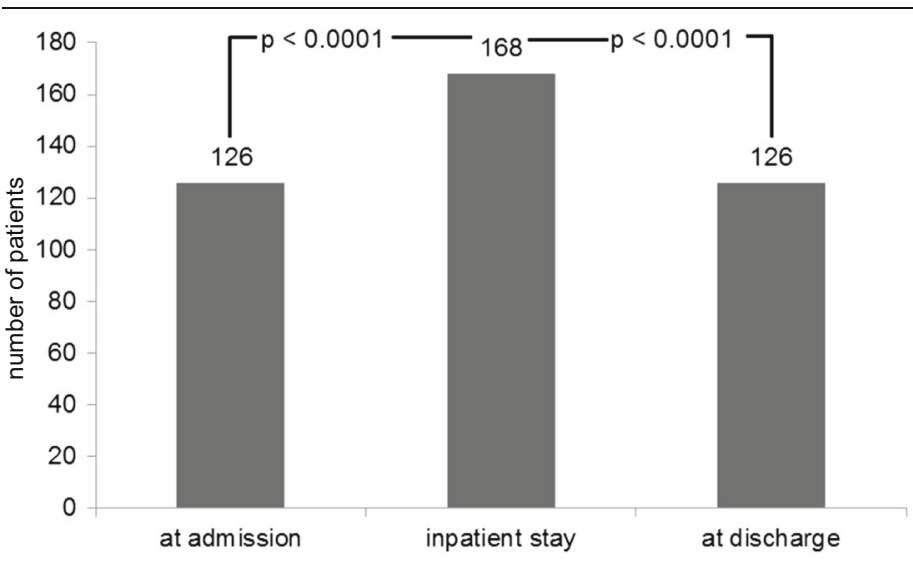

b Drug level

\begin{tabular}{llllll}
\hline & PIM (total) & FORTA & PRISCUS & STOPP \\
\hline At admission & $12.7 \%: 223$ of 1757 drugs & $98(44 \%)$ & $55(25 \%)$ & $163(73 \%)$ \\
\hline Inpatient stay & $11.9 \%: 397$ of 3324 drugs & $157(40 \%)$ & $161(41 \%)$ & $224(56 \%)$ \\
\hline At discharge & $10.5 \%: 217$ of 2068 drugs & $88(41 \%)$ & $58(27 \%)$ & $158(73 \%)$ \\
\hline
\end{tabular}

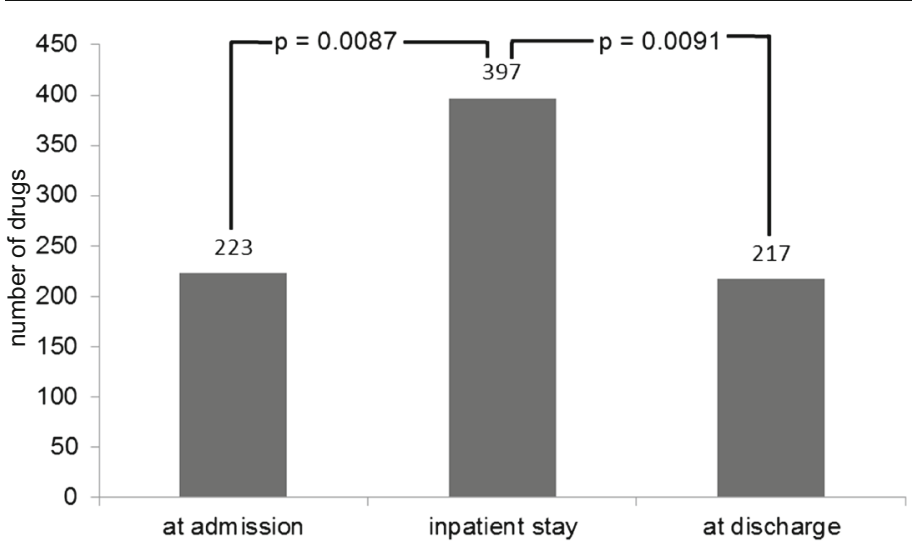

significant difference could be shown between admission and the inpatient stay $(P=0.0023)$.

When FORTA, PRISCUS and STOPP were compared with each other, significant differences in the numbers of PIMs could be shown at admission and at discharge between all sets of criteria at the patient level and at the drug level. During the inpatient stay, no significant difference concerning the numbers of PIMs was found between FORTA and PRISCUS (see Table 3).

Concerning individual PIMs, it can be stated that at admission, acetylsalicylic acid ( $N=19$; STOPP), followed by amlodipine $(N=15 ;$ STOPP $)$ and prednisolone ( $N=12$; FORTA and STOPP) were the PIMs prescribed most often. 
Table $3 P$ values for comparisons of the differences in prescribing of FORTA, PRISCUS and STOPP potentially inappropriate medications (PIMs) at admission, during the inpatient stay and at discharge

\begin{tabular}{llllllll}
\hline Stages & \multicolumn{1}{l}{$P$ values } & & & & \\
\cline { 2 - 3 } & Patient level & & & Drug level & & \\
\cline { 2 - 3 } \cline { 5 - 7 } & FORTA/PRISCUS & FORTA/STOPP & PRISCUS/STOPP & & FORTA/PRISCUS & FORTA/STOPP & PRISCUS/STOPP \\
\hline Admission & 0.0001 & 0.0176 & 0.0001 & 0.0001 & 0.0001 & 0.0001 \\
Inpatient stay & NS & 0.0490 & 0.0280 & NS & 0.0005 & 0.0003 \\
Discharge & 0.0011 & 0.0001 & 0.0001 & 0.0048 & 0.0001 & 0.0001 \\
\hline
\end{tabular}

FORTA (Fit for the Aged), NS not significant, PRISCUS [Latin for 'time-honoured'], STOPP (Screening Tool of Older Persons' Potentially Inappropriate Prescriptions)

Table 4 Numbers of patients receiving the three most frequently prescribed potentially inappropriate medication types (grouped according to level 3 Anatomical Therapeutic Classification codes) at admission, during the inpatient stay and at discharge

\begin{tabular}{llll}
\hline $\begin{array}{l}\text { Frequency of } \\
\text { prescription }\end{array}$ & Stages & & Discharge \\
\cline { 2 - 4 } & Admission & Inpatient stay & N05A: antipsychotics $(N=26)$ \\
\hline $\begin{array}{c}\text { st most } \\
\text { frequent }\end{array}$ & $\begin{array}{c}\text { B01A: antithrombotic } \\
\text { agents }(N=28)\end{array}$ & N05C: hypnotics and sedatives $(N=80)$ & C08C: selective calcium channel blockers with \\
mainly vascular effects $(N=24)$ \\
$\begin{array}{c}\text { frequent } \\
\begin{array}{c}\text { 3rd most } \\
\text { frequent }\end{array}\end{array}$ & $\begin{array}{c}\text { N05A: antipsychotics } \\
(N=26)\end{array}$ & N05A: antipsychotics $(N=39)$ & N06A: antidepressants $(N=23)$ \\
& $(N=20)$ & $\begin{array}{c}\text { C08C: selective calcium channel blockers with } \\
\text { mainly vascular effects }(N=33)\end{array}$ & \\
\hline
\end{tabular}

During the inpatient stay, zopiclone was classified as a PIM $(N=60$; PRISCUS) most often (at admission, $N=8$ only), followed by amlodipine ( $N=22$; STOPP) and lorazepam ( $N=17$; FORTA 17, STOPP 6 and PRISCUS 3$)$.

At discharge, amlodipine $(N=20)$, followed by acetylsalicylic acid $(N=16)$ and risperidone $(N=12)$, were the PIMs prescribed most often, all according to the STOPP rating, as was risperidone, according to the FORTA rating. Zopiclone ( $N=7$; PRISCUS) was ranked only eighth.

When grouped according to level 3 ATC codes, drugs classified as hypnotics and sedatives were by far the most commonly used $(N=84)$. At admission, they were prescribed less frequently $(N=13)$. Antithrombotic agents $(N=28 ; \quad$ STOPP 26 and PRISCUS 2), antipsychotics $(N=26$; STOPP 23, FORTA 19 and PRISCUS 5) and antidepressants $(N=20 ; \quad$ FORTA $15, \quad$ STOPP 8 and PRISCUS 7) were the PIMs prescribed most often at admission (see Table 4).

During the inpatient stay, hypnotics and sedatives were prescribed most often $(N=80$; PRISCUS 80, FORTA 11 and STOPP 7), followed by antipsychotics $(N=39$; FORTA 30, STOPP 30 and PRISCUS 6) and selective calcium channel blockers with mainly vascular effects $(N=33$; STOPP 30 and PRISCUS 6).

At discharge, drugs classified as antipsychotics were prescribed most often $(N=26$; STOPP 23, FORTA 19 and PRISCUS 3), followed by selective calcium channel blockers with mainly vascular effects $(N=24$; STOPP 23 and PRISCUS 2) and antidepressants $(N=23$; FORTA 18, STOPP 10 and PRISCUS 9).

Subgroup analyses exhibited significant differences in:

- STOPP concerning sex $(P=0.0324)$. More women were receiving STOPP PIMs.

- PRISCUS $(P=0.0435)$ and STOPP $(P=0.0384)$ concerning the previous residential situation. Patients living at home were prescribed more PIMs according to PRISCUS but fewer PIMs according to STOPP, in comparison with patients living in nursing homes.

- FORTA $(P=0.0034)$ and PRISCUS $(P<0.0001)$-as well as the total $(P=0.0092)$ - concerning the number of prescribed drugs. The more drugs were prescribed, the more PIMs were prescribed as well.

- PRISCUS $(P=0.0298)$ concerning the length of the hospital stay. A longer hospital stay was related to prescription of more PIMs.

- $\quad$ STOPP $(P=0.0010)$ concerning the hospital stay in a surgical ward versus an internal medicine ward. More STOPP PIMs were prescribed for patients staying in surgical wards.

\subsection{Secondary Objectives}

Is there a possible association between PIM use and the primary diagnosis/reason for admission, as well as severe 
side effects and consequences of PIM use during hospitalization?

Following an analysis using the WHO-UMC system for standardized case causality assessment, an association with the reason for admission could be found for amlodipine (in 10 of 15 patients) most often, followed by risperidone (in 6 of 9 patients), lorazepam (in 4 of 6 patients) and diclofenac (in 2 of 5 patients). These PIMs had severe side effects during the inpatient stay, such as falls following amlodipine, lorazepam and risperidone intake, and gastrointestinal bleeding following non-steroidal anti-inflammatory drug (NSAID) intake.

Applying both the results reported herein and an analysis of the available literature, a standardized workflow could be established to minimize the prescription of PIMs. This tool, designated as GERAS (Geriatrische Arzneimitteltherapie-Sicherheit [German for 'geriatric medication safety']), is used in routine clinical practice at UKE. GERAS consists of five items, is illustrated on just one page and is more a guidance for clinical situations than a list of single substances. For example, in item 4 ('Procedure for insomnia'), it is recommended that in patients aged over 65 years, the dosage of zopiclone should be limited to $3.75 \mathrm{mg}$. GERAS is provided in the Electronic Supplementary Material.

\section{Discussion}

In the present study, FORTA, PRISCUS and STOPP were applied simultaneously to one collective of patients for the first time. These three sets of criteria were chosen because they have been shown to be suitable for the German pharmaceutical market.

An overall PIM prevalence of $88 \%$ was found. In Germany, the PIM prevalence in elderly patients found in other investigations has ranged between 16 and $67 \%$ [10, 16-19]. Most studies have been conducted in the community/outpatient setting. Siebert et al. [10] found a PIM prevalence of $43 \%$ according to the PRISCUS rating and $67 \%$ according to the STOPP rating in a geriatric rehabilitation clinic. In particular, drugs from the group of hypnotics and sedatives, as well as antidepressants, have been identified as PIMs in many studies $[8,10,16]$. This is in accordance with the results of the present study. The reason for the observed high PIM prevalence might be that the study inclusion criteria included multimorbid patients receiving polypharmacy. In addition, if the PIM ratings according to FORTA, PRISCUS and STOPP are considered, it should be noted that the three sets of criteria rate drugs as PIMs quite differently. This also contributed to the high PIM prevalence in the present study. The intersections between the different sets of PIM criteria were quite small, which resulted in a greater number of medications being identified as PIMs.

Significant differences could be found at both the patient level and the drug level. Whereas PRISCUS primarily rates drugs from the group of hypnotics and sedatives as PIMs, FORTA and STOPP particularly rate antipsychotics and NSAIDs as PIMs. NSAIDs were striking in an investigation by Gallagher and O'Mahony [8] as well. Gallagher and O'Mahony [8] and Dormann et al. [17] also rated amlodipine as negative or as being associated with adverse events. In the present study, amlodipine was evaluated as a 'vasodilator drug' in accordance with STOPP and ranked as a PIM in all patients who were admitted to hospital because of a fall (even if postural hypotension could not always be identified as its cause), because of the retrospective design of the study. This possibly led to an increased negative rating but ensured a consistent rating within the study.

In the present study, a significant rise in use of PIMs during the inpatient stay was detected. A similar finding was also reported by Siebert et al. [10] and might be explained by the increased (temporary) use of hypnotics and sedatives during the inpatient stay. The increased use of potentially inappropriate analgesics during the inpatient stay also adds to this point.

The significant rise in PIM use from admission to the inpatient stay and the significant decrease from the inpatient stay to discharge illustrate that a reduction in prescribing of PIMs during the inpatient stay is essential, as it is known that avoiding PIM use in older adults is one strategy to decrease the risk of adverse events.

Reducing the total number of prescribed drugs might be a starting point, as a subgroup analysis of this study revealed that an increase in the number of prescribed drugs had a significant effect on the number of PIMs prescribed. Polypharmacy is a risk factor for PIM use. This has also been reported in several other studies [7, 9, 18, 20-28].

Concerning the causality between PIM use and the reason for admission, amlodipine and risperidone were the prevailing ones. These drugs were also associated with adverse events in the study by Dormann et al. [17]. As they used PRISCUS to identify PIMs, amlodipine and risperidone were rated as non-PIMs in that study. Concerning PRISCUS PIMs, acetyldigoxin, diazepam, tetrazepam and zolpidem were associated with falls in the study by Dormann et al. [17]. In the study by Gallagher and O'Mahony [8], which used the STOPP criteria, overt digoxin toxicity with high-dose digoxin and renal impairment, upper gastrointestinal bleeding with inappropriate NSAID use, and falls with inappropriate psychotropic use could be identified as causal or contributory to admission. In the present study, NSAIDs were also found to be potentially causal for admission. 
During the inpatient stay, use of lorazepam, risperidone, mirtazapine, zopiclone and dimenhydrinate was associated with falls. Therefore, hypnotics and sedatives seem to be of particular importance.

Nevertheless, it has to be stated that PIMs are only potentially (not definitely) inappropriate, and that detailed clinical judgment is always needed, based on a full review of the medical record.

\subsection{Comparison of FORTA, PRISCUS and STOPP}

Negative lists such as PRISCUS, which provide an explicit listing of drugs, independent of the diagnosis, are easy to use - the main advantage being good practicability. On the other hand, constant updates are needed, and such lists carry the risk of an assumption that drugs not listed would be appropriate in every case. As an example, the NSAID indometacin is listed, but diclofenac is not. In particular, the STOPP criteria have the advantage of greater flexibility due to a waiver of indexing certain drugs, so international transferability is assured, and an adaption to current market conditions is not necessary. In addition, contraindications and duplicate prescriptions can also be identified using STOPP. As a critical note regarding STOPP, it can be stated that use of the criteria is sophisticated, and a gradual rating is not possible. FORTA allows a disease-related evaluation revealing over-treatment and under-treatment, and substances rated negatively as class D can be replaced by more suitable substances in class A or class B.

The three sets of criteria have in common that they refer to long-term medication. Drugs frequently used during the inpatient stay, such as antibiotics, are hardly taken into account. The only antibiotic listed by PRISCUS is nitrofurantoin.

The superiority of more complex criteria, such as STOPP, to negative lists has already been demonstrated by Hamilton et al. [29] with respect to adverse events and resulting hospital admissions.

In the present study, in 176 of 200 patients, at least one PIM in their medication was found: in 116 patients according to FORTA, in 113 patients according to PRISCUS and in 138 patients according to STOPP. So, the largest number of PIMs were identified by STOPP. It is striking that medication is rated quite differently by the three sets of criteria. A comparison between FORTA, PRISCUS and STOPP showed that there are significant differences between them. Thus, the present study confirms the superiority of STOPP to purely negative lists, as has also previously been shown by Gallagher and O'Mahony [8] and Hamilton et al. [29], who compared the STOPP and Beers criteria.

Looking at PIM use related to the reason for admission, FORTA identified 30 possible and 50 unlikely relationships, PRISCUS identified 9 possible and 19 unlikely relationships, and STOPP identified 50 possible and 66 unlikely relationships. In percentage terms, FORTA identified $38 \%$ possible PIM-adverse drug event associations, PRISCUS identified $32 \%$ and STOPP identified $43 \%$.

With the implementation of the new practical tool GERAS in routine clinical practice at UKE, the rate of zopiclone $7.5 \mathrm{mg}$ being the most frequently prescribed PIM in the present study could be reduced from 30 to $7 \%$ (unpublished results).

\subsection{Limitations}

Because of the retrospective design of the study, only events documented in the patient record were considered, so the rate of adverse events might have been higher in reality. In addition, only possible associations following the WHO-UMC causality categories of PIMs and adverse events could be stated. Moreover, only the medication documented in the electronic patient record could be considered. The study population was quite small, and so larger, randomized, multicentre studies are needed to confirm the findings of the present study.

\section{Conclusion}

The three sets of criteria-FORTA, PRISCUS and STOPP_rate drugs as PIMs quite differently. A comparison between FORTA, PRISCUS and STOPP showed that there are significant differences between them. In addition, especially during the inpatient stay, prescribing of PIMs was shown to be common. As it is known that avoiding PIM use in older adults is one strategy to decrease the risk of adverse events, a reduction in PIM use during the inpatient stay is essential. Polypharmacy was identified as a risk factor for PIM use, and so reducing the total number of prescribed drugs might be a starting point to reduce PIM use.

\section{Compliance with Ethical Standards}

Conflicts of Interest Beate Wickop, Steffen Härterich, Christian Sommer, Anne Daubmann, Michael Baehr and Claudia Langebrake declare no conflict of interest.

Funding No funding was received for this work.

Ethical Approval Ethical approval was obtained from Ethikkommission der Ärztekammer Hamburg.

Open Access This article is distributed under the terms of the Creative Commons Attribution-NonCommercial 4.0 International License (http://creativecommons.org/licenses/by-nc/4.0/), which permits any noncommercial use, distribution, and reproduction in any medium, provided you give appropriate credit to the original author(s) and the source, provide a link to the Creative Commons license, and indicate if changes were made. 


\section{References}

1. Mangoni AA, Jackson SHD. Age-related changes in pharmacokinetics and pharmacodynamics: basic principles and practical applications. Br J Clin Pharmacol. 2004;57(1):6-14.

2. Tinetti ME. Preventing falls in elderly persons. N Engl J Med. 2003;348(1):42-9.

3. Blanco-Reina E, Ariza-Zafra G, Ocaña-Riola R, León-Ortiz M. 2012 American Geriatrics Society Beers criteria: enhanced applicability for detecting potentially inappropriate medications in European older adults? A comparison with the Screening Tool of Older Person's Potentially Inappropriate Prescriptions. J Am Geriatr Soc. 2014;62(7):1217-23.

4. Wehling M, Burkhardt H. Arzneitherapie für Ältere. 2nd ed. Berlin: Springer; 2011.

5. Holt S, Schmiedl S, Thürmann PA. Potentially inappropriate medications in the elderly: the PRISCUS list. Dtsch Ärztebl Int. 2010;107(31-32):543-51.

6. Gallagher P, Ryan C, Byrne S, Kennedy J, O'Mahony D. STOPP (Screening Tool of Older Person's Prescriptions) and START (Screening Tool to Alert Doctors to Right Treatment): consensus validation. Int J Clin Pharmacol Ther. 2008;46(2):72-83.

7. Gallagher P, Lang PO, Cherubini A, et al. Prevalence of potentially inappropriate prescribing in an acutely ill population of older patients admitted to six European hospitals. Eur J Clin Pharmacol. 2011;67(11):1175-88.

8. Gallagher P, O'Mahony D. STOPP (Screening Tool of Older Persons' Potentially Inappropriate Prescriptions): application to acutely ill elderly patients and comparison with Beers' criteria. Age Ageing. 2008;37(6):673-9.

9. McMahon CG, Cahir CA, Kenny RA, Bennett K. Inappropriate prescribing in older fallers presenting to an Irish emergency department. Age Ageing. 2014;43(1):44-50.

10. Siebert S, Elkeles B, Hempel G, Kruse J, Smollich M. The PRISCUS list in clinical routine: practicability and comparison to international PIM lists. Z Gerontol Geriatr. 2012;46(1):35-47.

11. Morin L, Fastbom J, Laroche M-L, Johnell K. Potentially inappropriate drug use in older people: a nationwide comparison of different explicit criteria for population-based estimates. Br J Clin Pharmacol. 2015;80(2):315-24.

12. WHO Collaborating Centre for Drug Statistics Methodology. ATC/DDD index. http://www.whocc.no/atc_ddd_index. Accessed 7 Dec 2015.

13. Deutsches Institut für Medizinische Dokumentation und Information. Das DIMDI-Medizinwissen online. http://www.dimdi. de. Accessed 4 May 2014.

14. Kuhn-Thiel AM, Weiss C, Wehling M, FORTA Authors/Expert Panel Members. Consensus validation of the FORTA (Fit for the Aged) list: a clinical tool for increasing the appropriateness of pharmacotherapy in the elderly. Drugs Aging. 2014;31(2): $131-40$.

15. Uppsala Monitoring Centre. The use of the WHO-UMC system for standardised case causality assessment. http://who-umc.org/ Graphics/24734.pdf. Accessed 5 May 2014.
16. Amann U, Schmedt N, Garbe E. Prescribing of potentially inappropriate medications for the elderly: an analysis based on the PRISCUS list. Dtsch Ärztebl Int. 2012;109(5):69-75.

17. Dormann H, Sonst A, Müller F, et al. Adverse drug events in older patients admitted as an emergency: the role of potentially inappropriate medication in elderly people (PRISCUS). Dtsch Ärztebl Int. 2013;110(13):213-9.

18. Schubert I, Küpper-Nybelen J, Ihle P, Thürmann P. Prescribing potentially inappropriate medication (PIM) in Germany's elderly as indicated by the PRISCUS list: an analysis based on regional claims data. Pharmacoepidemiol Drug Saf. 2013;22(7):719-27.

19. Fiss T, Dreier A, Meinke C, van den Berg N, Ritter CA, Hoffmann W. Frequency of inappropriate drugs in primary care: analysis of a sample of immobile patients who received periodic home visits. Age Ageing. 2011;40(1):66-73.

20. Bradley MC, Fahey T, Cahir C, et al. Potentially inappropriate prescribing and cost outcomes for older people: a cross-sectional study using the Northern Ireland Enhanced Prescribing Database. Eur J Clin Pharmacol. 2012;68(10):1425-33.

21. Cahir C, Fahey T, Teeling M, Teljeur C, Feely J, Bennett K. Potentially inappropriate prescribing and cost outcomes for older people: a national population study. $\mathrm{Br} \mathrm{J}$ Clin Pharmacol. 2010;69(5):543-52.

22. Frankenthal D, Lerman Y, Kalendaryev E, Lerman Y. Potentially inappropriate prescribing among older residents in a geriatric hospital in Israel. Int J Clin Pharm. 2013;35(5):677-82.

23. Galvin R, Moriarty F, Cousins G, et al. Prevalence of potentially inappropriate prescribing and prescribing omissions in older Irish adults: findings from the Irish Longitudinal Study on Ageing study (TILDA). Eur J Clin Pharmacol. 2014;70(5):599-606.

24. O'Sullivan DP, O'Mahony D, Parsons C, et al. A prevalence study of potentially inappropriate prescribing in Irish long-term care residents. Drugs Aging. 2013;30(1):39-49.

25. Onatade R, Auyeung V, Scutt G, Fernando J. Potentially inappropriate prescribing in patients on admission and discharge from an older peoples' unit of an acute UK hospital. Drugs Aging. 2013;30(9):729-37.

26. Vishwas HN, Harugeri A, Parthasarathi G, Ramesh M. Potentially inappropriate medication use in Indian elderly: comparison of Beers' criteria and Screening Tool of Older Persons' Potentially Inappropriate Prescriptions. Geriatr Gerontol Int. 2012; 12(3):506-14.

27. Wahab MSA, Nyfort-Hansen K, Kowalski SR. Inappropriate prescribing in hospitalised Australian elderly as determined by the STOPP criteria. Int J Clin Pharm. 2012;34(6):855-62.

28. Ryan C, O'Mahony D, Kennedy J, et al. Potentially inappropriate prescribing in older residents in Irish nursing homes. Age Ageing. 2013;42(1):116-20.

29. Hamilton H, Gallagher P, Ryan C, Byrne S, O'Mahony D. Potentially inappropriate medications defined by STOPP criteria and the risk of adverse drug events in older hospitalized patients. Arch Intern Med. 2011;171(11):1013-9. 